\title{
Parameterryke onvoorwaardelike verdelings met 'n stogastiese parameter in die voorwaardelike gammaverdeling
}

\author{
A. Bekker en J. J. J. Roux* \\ Departement Statistiek, Universiteit van Suid-Afrika, \\ Posbus 392, Pretoria 0001
}

Ontrang 17 Jumie 1991; aumvarr 23 Augusrus 1991

\section{UITTREKSEI.}

Hier word veronderstel dat die skaalparameter in die tweeparameter-gammaverdeling (as voonwardelike verdeling) 'n funksie van 'n variaat is. Hierdie stogastiese skaalparameter nord hepal dear die vorm wat die regressiekromme kan anneem. Vir die regressiefunksies ay en $\frac{a}{y}$ word parametervke onvoorwardelike verdelings met die weeparametergammaverdeling as vermengingsverdeling afgelei. in Veralgemeende verdeling word as vermengingsverdeling gehruik om in we klas van onvoorwadelike verdelings af te lei, met verryking teweeggehring deur regressieparameters.

\begin{abstract}
Parameter-rich unconditional distributions with a stochastic parameter in the conditional gamma distribution

Suppose that the scale parameter in the wo-parameter gamma distribution (as conditional distribution) is a function of a variate. This stochastic scale parameter is determined hy the form which the regression curve can assume. Parameter-rich unconditional distributions with the wo-parameter gamma distribution as a mixed distribution are derived for the regression functions ay and $\frac{a}{y}$. A generalized distribution is used as a mixed distrihution to derive a wide class of unconditional distrihutions with enrichment induced by regression parameters.
\end{abstract}

*Outeur aan wie korrespondensie gerig kan word. 


\section{INLEIDING}

Veronderstel dat die skaalparameter, 0 , in die tweeparameter-gammaverdeling afhanklik van 'n variaat $Y$ is. Die voorwaardelike waarskynlikheidsdigtheidsfunksie (wdf.) van $X$ gegee $Y=y$, word dus gegee deur

(1.1) $f(x \mid y)=\frac{x^{x} e_{e} x(y)}{(\theta(y))^{x} \Gamma(x)}$

$$
(x>0, \theta(1)>0, x>0)
$$

en aangedui deur $\mathrm{X} \mid \mathrm{Y} \sim \mathrm{Gtp}(\theta(1), x)$.

Vir wdf. (1.1) is die regressiefunksie $\mathrm{E}(\mathrm{X} \mid \mathrm{Y})=(0)()$. waar die waarde van $\theta(y)$ bepaal word deur die vorm ay of $\frac{a}{y}$ wat die regressiek romme kan aanneem. (Let op dat algebraïese redes die keuse van die regressiefunksie bepaal.)

Hier is die variatat $Y$ ' $n$ faktor wat die verdeling van $\mathrm{X}$ verander. ${ }^{.}$Die onvoorwaardelike wdf. van $\mathrm{X}$ volg vanuit

(1.2) $h(x)=\int f(x \mid y) g(y) d y$

vir 'n gegewe wdf, van $Y$ (die verdeling van $Y$ word ook die vermengingsverdeling genoem). Let ook op dat hierdie onvoorwaardelike verdeling minstens een meer parameter, naamlik die regressieparameter (regressiekoëffisiënt) $a$, as die bekende verdeling besit. Hierdie afhanklikheidsparameter kan as 'n ongelykheidsfaktor geinterpreteer word. Ter verduideliking van laasgenoemde: veronderstel dat $f(x \mid y)$ die wdf. van die voorwaardelike inkomsteverdeling is met variaat $Y$ ' $n$ inkomstebepalende faktor, sê ouderdom. Figuur 1 illustreer dat vir 'n gegewe ouderdom $y$ ' die verwagte inkomste van 'n onderwyser $x$ ' is, teenoor die verwagte inkomste $x$ " van "n algemene praktisyn.

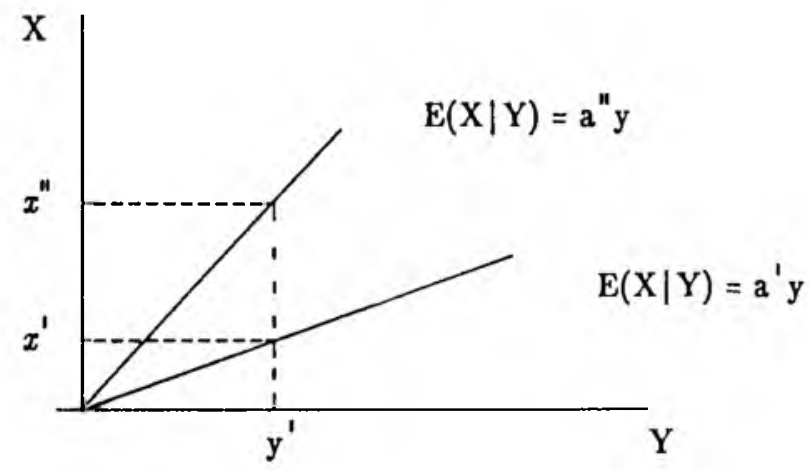

FIGUUR 1: Die rol van die regressieparameter a in $E(X \mid Y)=a y$.

Vervolgens word in \$2 parameterryke onvoorwaardelike verdelings met die tweeparameter-gammaverdeling as die vermengingsverdeling en die regressiefunksies $a y$, en $\frac{a}{y}$ afgelei.

In plaas daarvan om telkens die onvoorwaardelike verdeling vir verskillende tipes vermengingsverdelings te verkry, word nou in $\$ 3$ veronderstel dat variaat $Y^{\prime} n$ veralgemeende verdeling, naamlik die G-funksieverdeling, besit en dat ' $n$ algemene tipe regressiefunksie $a y^{\prime}$ van toepassing is. Die belangrikheid van die G-funk$\mathrm{sie}^{2}$ is die feit dat byna al die spesiale funksies in toegepaste wiskunde en statistiek spesiale gevalle daarvan is. 'n Wye klas van onvoorwaardelike verdelings volg. met verryking teweeggebring deur die regressieparameters $a$ en $b$. Deur die toewysing van sekere waardes aan die parameters van die regressiefunksie en die vermengingsverdeling kan die onvoorwaardelike verdelings bepaal word, waarvan enkele gevalle in tabel 1 getoon word.

\section{AFLEIDINGS}

\section{RESULTAAT 2.1}

Veronderstel dat $X \mid Y \sim G \operatorname{tp}(\theta(v), x)$ (die lokaliteitsparameter 'n funksie van $Y$ ), $Y \sim \operatorname{Gtp}\left(\frac{1}{\gamma}, \delta\right)$ en $\mathrm{E}(\mathrm{X} \mid \mathrm{Y})=a$; dan besit $\mathrm{X}$ die saamgestelde gammaverdeling van die tweede soort met parameters $\frac{a}{\gamma \alpha}, \delta$ en $x$.

\section{BEWYS}

Vanuit $E(X \mid Y)=\theta(y) x$ en die spesifikasie dat $\mathrm{E}(\mathrm{X} \mid \mathrm{Y})=a y$, volg dat $\theta(y)=\frac{a y}{\alpha}$. Die onvoorwaardelike wdf. van $X$ word gegee deur

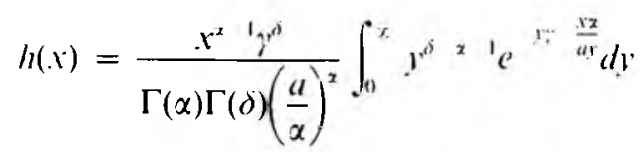

waar

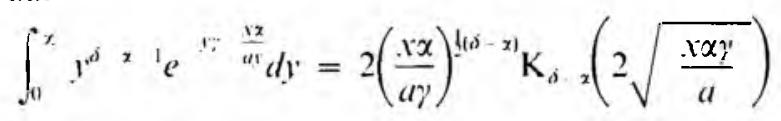

(Erdélyi, et al., p. 313).

Gevolglik is

$$
\begin{aligned}
& h(x)=2 {\left[\frac{x}{\frac{a}{x_{\gamma}^{\prime}}}\right]^{\lfloor(x+\delta)-1} } \\
&\left.\cdot \mathrm{K}_{\delta}\left(\sqrt[2]{\frac{x \alpha_{i}^{\prime}}{a}}\right) / \Gamma(x) \Gamma(\delta)\left(\frac{a}{x \gamma}\right)\right] \\
& \quad(x>0, a>0, \gamma>0, \delta>0, x>0) .
\end{aligned}
$$

\section{OPMERKING 2.1}

(i) Die saamgestelde gammaverdeling van die tweede soort soos in (2.1) gegee, het dieselfde vorm as die verdeling wat deur Bhattacharya ${ }^{4}$ ondersoek is,

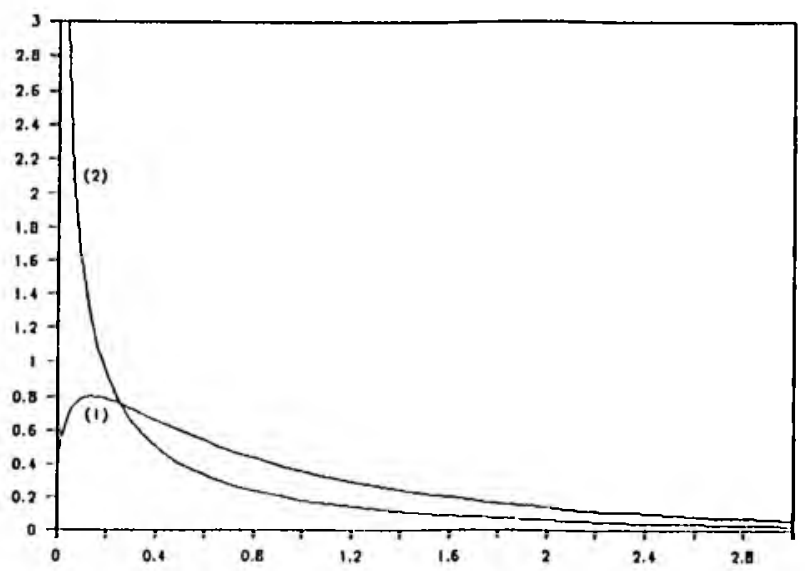

FIGUUR 2: Die wdf' 's van die saamgestelde gammaverdeling van die weede soort vir $a=1 ; \gamma=2$ en (1) $\alpha=1,5 ; \delta=2,5$ en (2) $\alpha=0,5 ; \delta=1,5$. 
maar met 'n ekstra parameter, naamlik die regressieparameter $a$. (Let on dat vir $a=\alpha$ reduseer (2. I) na vgl. (33) van Bhattacharya ${ }^{-1}$ met $\frac{1}{\gamma}=\beta$.)

(ii) Die h-de moment van $X$ mel wdf. (2.1) is

$$
\mathrm{E}(\mathrm{X})^{\prime \prime}=\frac{\Gamma(x+h) \Gamma(\delta+h)}{\Gamma(x) \Gamma(\delta)}\left(\frac{a}{x \gamma}\right)^{\prime \prime} .
$$

(iii) Figuur 2 illustreer die krommevorme van wdf. (2.1) vir verskillende waardes van die parameters $x$ en $\delta$, maar vir vaste waardes van $a$ en $\gamma$. Hier moel egter daarop gelet word dat die uitdrukkings vir die maatstal van kurtose $\left(\frac{\mu_{4}}{\mu_{2}^{2}}-3\right)$ en die maatstal van skeetheid $\left(\frac{\mu_{3}}{\mu_{3}^{\frac{3}{3}}}\right)$ (vir $\left.\mu_{h}=\mathrm{E}(\mathrm{X}-\mathrm{E}(\mathrm{X}))^{h}, h=0,1,2, \ldots\right)$ onalhanklik van die parameters $a$ en $\gamma$ is.

(iv) Daar kan van die relasie tussen die $h$-de moment (om die nulpumt) van die saamgestelde verdeling

TABEI, 1

Onvoorwaardelike verdelings wat betrekking het op die gevalle $\mathrm{E}(\mathrm{X} \mid \mathrm{Y})=a y, \mathrm{E}(\mathbf{X} \mid \mathrm{Y})=\frac{a}{y}$ en $\mathrm{E}(\mathrm{X} \mid \mathrm{Y})=a y^{2}$

\begin{tabular}{|c|c|c|c|c|c|c|}
\hline Randverdeling van $Y$ & $k i r s$ & K & $a_{1}$ & b) & $\begin{array}{l}\text { Parameters } \\
\text { van dic } \\
\text { regressie- } \\
\text { lunksic } \\
a b \quad b\end{array}$ & Onvoorwaardelike verdeling van $\mathrm{X}$ \\
\hline $\begin{array}{l}\text { (1) Tweeparameler-gamma } \\
\begin{array}{l}y^{\prime \prime} \text { 'e } \frac{y^{\prime}}{\Gamma(\delta)} \\
\qquad(y>0 . \delta>0, ;>0)\end{array}\end{array}$ & $\begin{array}{llll}1 & 0 & 0 & 1\end{array}$ & $\frac{i}{\Gamma(\delta)}$ & - & $(\delta-1)$ & 1 & 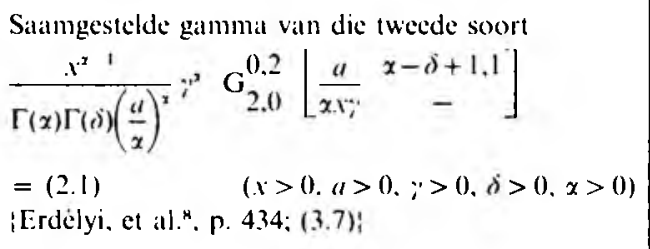 \\
\hline $\begin{array}{l}\text { (2) Tweeparameter-gatmma } \\
\qquad \begin{array}{l}\frac{y^{\prime}}{\Gamma(\delta)} \\
(y>0, \delta>0, y>0)\end{array}\end{array}$ & $1 \begin{array}{llll}1 & 0 & 0 & 1\end{array}$ & $\frac{\ddot{i}}{\Gamma(\dot{\delta})}$ & - & $(\delta-1)$ & -1 & 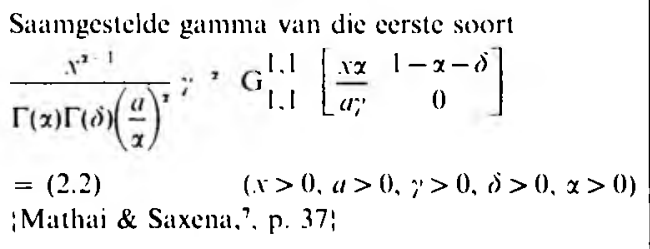 \\
\hline $\begin{array}{l}\text { (3) Tweeparameter-gamma } \\
\qquad \begin{array}{l}\frac{y^{\prime}}{\Gamma(\delta)} \\
(y>0, \delta>0, y>0)\end{array}\end{array}$ & 100101 & $\frac{i}{\Gamma(\delta)}$ & - & $(\delta-1)$ & 2 & 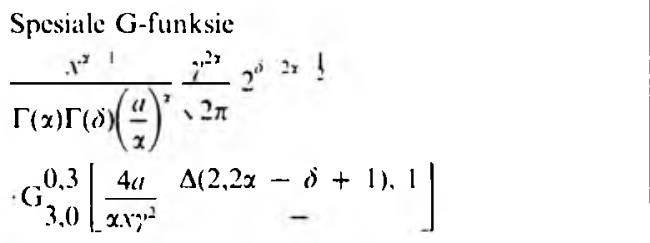 \\
\hline $\begin{array}{l}\text { (4) Standaardbeta } \\
\frac{y^{\prime \prime \prime}(1-y)^{\prime \prime}}{\mathrm{B}(m+n)} \\
(0<y<1, m>0, n>0)\end{array}$ & $\begin{array}{llll}1 & 0 & 1 & 1\end{array}$ & $\frac{\Gamma(m+n)}{\Gamma(m)}$ & $(m+n-1)$ & $(m-1)$ & -1 & 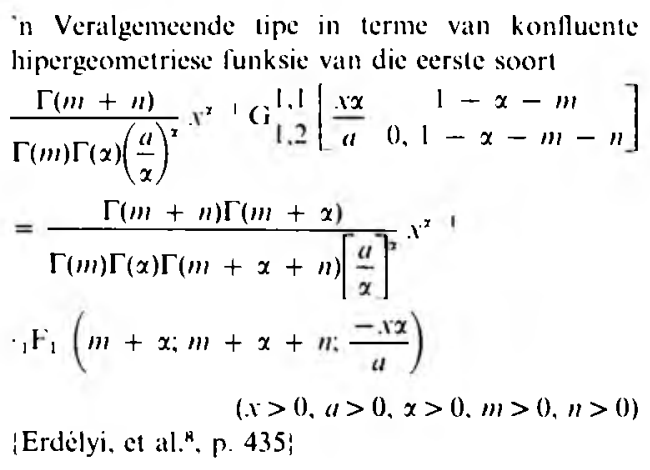 \\
\hline $\begin{array}{l}\text { (5) Beta van die tweede } \\
\text { soort } \\
\frac{f^{m} 1(1+1)^{(m+m)}}{\mathrm{B}(m, n)} \\
(1>0, m>0, n>0)\end{array}$ & 1111 & $\frac{1}{\Gamma(m) \Gamma(n)}$ & $(-n)$ & $(m-1)$ & 1 & 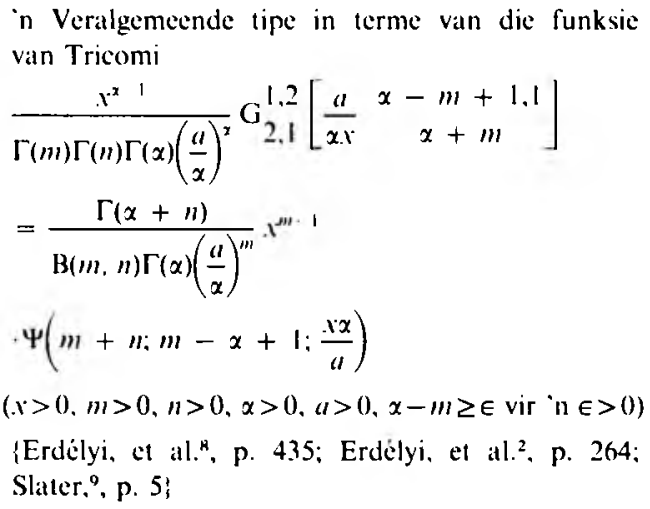 \\
\hline
\end{tabular}


en die $h$-de moment (om die nulpunt) van die vermengingsverdelings gebruik gemaak word om momentberamers vir die parameters van wdf. (2.1) te verkry, nalamlik

$$
\begin{aligned}
\mu_{h}(x) & =\int_{0}^{s} x^{\prime \prime} h(x) d x \\
& =\int_{0}^{\infty} \int_{0}^{s} x^{\prime \prime} f(x \mid y) g(y) d x d y \\
& =\frac{\Gamma(x+h)}{\Gamma(\alpha) \Gamma(\delta)}\left(\frac{a}{x}\right)^{h} \gamma^{\delta} \int_{0}^{\prime} y^{\prime \prime+h ~ e^{\prime \prime}} d y \\
& =\frac{\Gamma(\alpha+h)}{\Gamma(\alpha)}\left(\frac{a}{\alpha}\right)^{h} \mu_{h}(y) \text { waar } \\
\mu_{h}(y) & =\mathrm{E}(\mathrm{Y})^{h}=\frac{\Gamma(\delta+h)}{\Gamma(\delta) \gamma^{h} .}
\end{aligned}
$$

Definieer die steekproefmoment as

$$
u_{h}=\frac{1}{\mathrm{~N}} \sum_{i=1}^{\mathrm{N}} x_{i}^{\prime \prime} \text { en } v_{h}=\frac{1}{\mathrm{~N}} \sum_{i=1}^{\mathrm{N}} y_{i}^{\prime \prime}
$$

Aangesien $\quad \mu_{1}(v)=\hat{d} \hat{\gamma}=v_{1}^{\prime}$

$$
\begin{aligned}
\text { en } \mu_{2}(v) & =\hat{\delta}(\hat{\delta}+1) \hat{\gamma}^{2}=v_{2}, \\
\text { volg dat } \hat{\delta} & =\left[v_{2} /\left(v_{1}\right)^{2}-1\right] \\
\text { en } \hat{\gamma} & =v_{1}\left[v_{2} /\left(v_{1}\right)^{2}-1\right] .
\end{aligned}
$$

Hieruit en deur bostaande relasie te gebruik, word verkry dat

$$
\hat{a}=u_{1} / v_{1} \text { en } \hat{\alpha}=\left[u_{2}\left(v_{1}\right)^{2} /\left(u_{1}\right)^{2} v_{2}^{\prime}-1\right] \text { '. }
$$

\section{RESULTAAT 2.2}

Veronderstel dat $\mathrm{X} \mid \mathrm{Y} \sim \mathrm{Gtp}(0(\mathrm{y}), \alpha)$, $\mathrm{Y} \sim \mathrm{Gtp}\left(\frac{1}{\gamma}, \delta\right)$ en $\mathrm{E}(\mathrm{X} \mid \mathrm{Y})=\frac{a}{y}$; dan besit $\mathrm{X}$ die saamgestelde gammaverdeling van die eerste soort met parameters $\frac{a \gamma}{\alpha}, x$ en $\delta$.

\section{BEWYS}

Vanuit $\mathrm{E}(\mathrm{X} \mid \mathrm{Y})=\theta(\mathrm{y}) \alpha=\frac{a}{y} \operatorname{volg}$ dat $\theta(y)=\frac{a}{\alpha y}$ en is die onvoorwaardelike wdf. van $\mathrm{X}$ soos volg:

$$
\begin{aligned}
h(x)= & \frac{x^{x} \frac{1}{y^{\prime}}}{\Gamma(\alpha) \Gamma(\delta)\left(\frac{a}{x}\right)^{x}} \int_{0}^{x} y^{x+\delta \cdot 1} e^{r\left(y+\frac{x y}{a}\right)} d y \\
= & x^{x}\left(\frac{\alpha}{a \gamma}\right)^{x}\left(1+\frac{x \alpha}{a \gamma}\right)^{(x+\delta)} / \mathrm{B}(\alpha, \delta) \\
& (x>0, a>0, \delta>0, \gamma>0, x>0) .
\end{aligned}
$$

\section{OPMERKING 2.2}

(i) Indien $a=\alpha$, dan reduseer (2.2) na die saamgestelde wdf. soos deur Dubey ${ }^{0}$ afgelei.

(ii) Die $h$-de moment van $\mathrm{X}$ met wdf. (2.2) word gegee deur

$$
\mathrm{E}(\mathrm{X})^{h}=\left(\frac{a \gamma}{\alpha}\right)^{\prime \prime} \frac{\mathrm{B}(\alpha+h, \delta-h)}{\mathrm{B}(\alpha, \delta)}
$$

(iii) Figuur 3 toon die invloed van regressieparameter $a$ op die vorm van die onvoorwaardelike verdeling van $\mathrm{X}$ met wdf. (2.2) vir vaste waardes van $\alpha$, $\delta$ en $\gamma$. Hierdie saamgestelde gammaverdeling van die eerste soort sluit 'n verskeidenheid van krommevorme in, soos geillustreer deur die stip van wdf. (2.2) in figuur 4 vir verskillende waardes van $x$ en $\delta$, maar vir vaste waardes van $a$ en $\gamma$. (iv) Vir $\delta$ en $\gamma$ bekend, is die momentberamers vir $a$ en $\alpha$ die volgende:

$$
\begin{aligned}
& \hat{a}=u_{1} \frac{(\delta-1)}{\gamma} \text { en } \\
& \hat{\alpha}=\left[\frac{u_{2}(\delta-2)}{\left(u_{1}\right)^{2}(\delta-1)}-1\right]^{\prime} \\
& \text { met } \quad u_{h}^{\prime}=\frac{1}{N} \sum_{i=1}^{N} x_{i}^{h} \quad \text { die } \quad h \text {-de-orde }
\end{aligned}
$$
steekproefmoment.

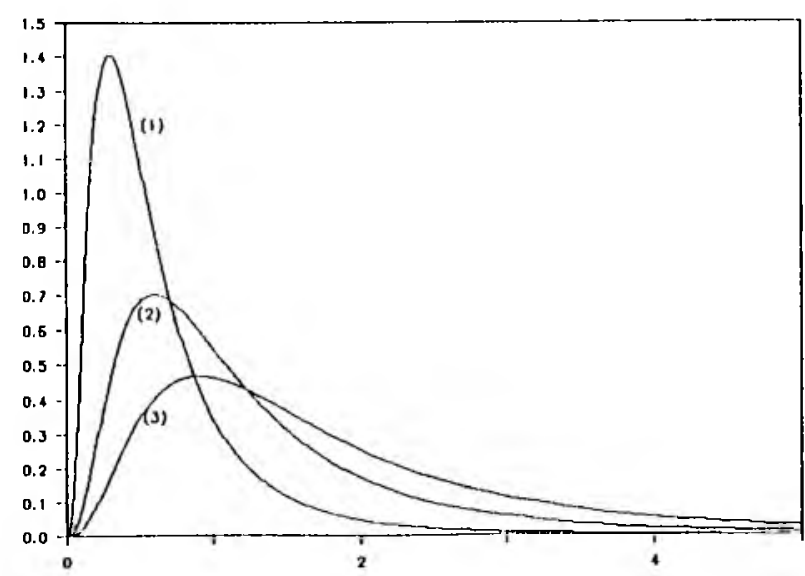

FIGUUR 3: Die w'df.'s van die saamgstelde gammaverdeling van die eerste soort vir $\alpha=4 ; \delta=4 ; \gamma=1$ en (1) $a=2$, (2) $a=4$ en (3) $a=6$.

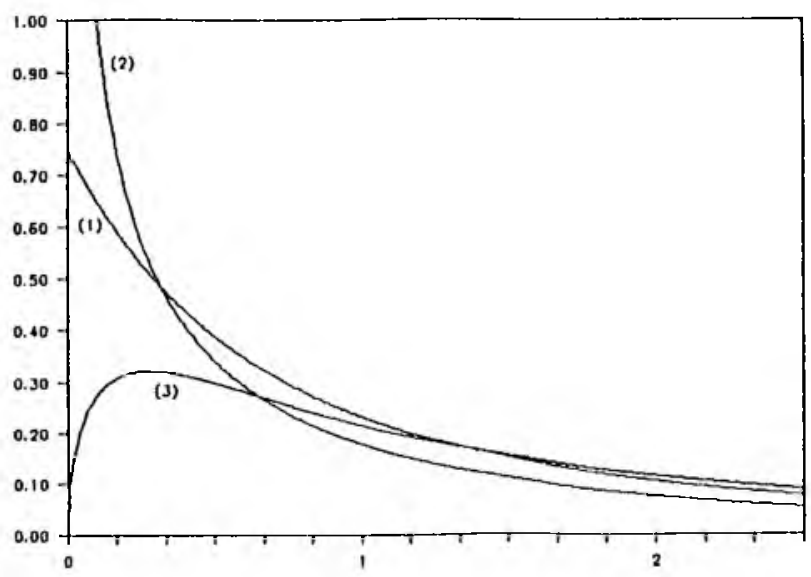

FIGUUR 4: Die 1'df.'s van die saamgestelde gammaverdeling van die eerste soort vir $a=1 ; \gamma=2$ en (1) $x=1 ; \delta=1,5$, (2) $\alpha=0,5 ; \delta=2$ en (3) $\alpha=1,5$;

$$
\delta=1 \text {. }
$$

\section{ALGEMENE RESULTAAT}

Veronderstel dat $\mathrm{X} \mid \mathrm{Y} \sim \operatorname{Gtp}(\theta(y), \alpha), \mathrm{E}(\mathrm{X} \mid \mathrm{Y})=a y^{h}$ $(a>0)$ en $\mathrm{Y}$ besit die G-funksieverdeling met wdf.

$$
g(y)=\mathrm{KG}_{r, s}^{k, 1}\left[\begin{array}{l|l}
\gamma y & a_{1}, \ldots, a_{r} \\
& b_{1}, \ldots, b_{s}
\end{array}\right]
$$

waar $\mathrm{G}(\gamma y)$ die G-funksie soos in Erdélyi, et al. ${ }^{2}$, p. 207 gedefinieer, voorstel; 
$\begin{aligned} \text { (3.2) } \mathrm{K}= & \gamma\left[\prod_{i=k+1}^{s} \Gamma\left(-b_{j}\right) \prod_{i=1}^{r} \Gamma\left(1+a_{j}\right)\right] \\ & \div\left[\prod_{i=1}^{k} \Gamma\left(1+b_{j}\right) \prod_{j=1}^{1} \Gamma\left(-a_{j}\right)\right]\end{aligned}$

die waardes van $\gamma, a,(j=1, \ldots, r), h,(j=1, \ldots, s)$ sodanig is dat $\int_{0}^{x} g(y) d y=1$ met $g(y) \geq 0$ vir r $>0$; en $\mathrm{S}$ die deelversameling van positiewe reële getalle is waarvoor $\mathrm{G}(\cdot)$ konvergeer.

Dan is die onvoorwaardelike wdf. van $\mathrm{X}$ soos volg:

$$
h(x)=\frac{\mathrm{K} x^{x}{ }^{1} \gamma^{h x} 1}{\Gamma(\alpha)\left(\frac{a}{\alpha}\right)^{x}}
$$

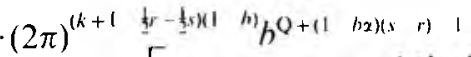

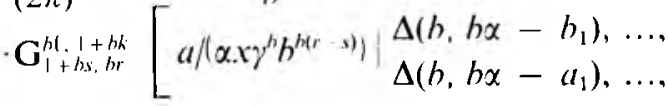

$\left.\begin{array}{l}\Delta\left(b, b \alpha-b_{s}\right), 1 \\ \Delta\left(b, b \alpha-a_{t}\right)\end{array}\right]$

(3.4) $=\frac{\mathrm{K} x^{x-1} \gamma^{h x} \quad 1}{\Gamma(\alpha)\left(\frac{a}{\alpha}\right)^{x}}$

$\cdot(2 \pi)^{\left(h+1-\frac{1}{r} r-\frac{1}{2}\right)(1+b)}(-b)^{Q+(1) h x)(s-r)-1}$

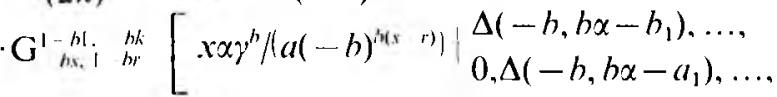

$\left.\begin{array}{l}\Delta\left(-b, h \alpha-b_{s}\right) \\ \Delta\left(-b, b \alpha-a_{1}\right)\end{array}\right]$

$(h>0)$

waar $\mathrm{K}$ in (3.2) gedefinieer word;

$(b<0)$

$$
\mathrm{Q}=\sum_{i=1}^{s} b_{i}-\sum_{i=1}^{r} a_{j}+\frac{1}{2} r-\frac{1}{2} s+1 ;
$$

$\Delta(b, t)$ die volgende versameling met $b$-parameters voorstel

$$
\frac{1}{b}, \frac{t+1}{h}, \ldots, \frac{t+h-1}{b} \text {; en }
$$

die waardes van $\alpha, a, b, \gamma, a_{i}(j=1, \ldots, r)$ en $b$, $(j=1, \ldots, s)$ sodanig is dat $h(x)$ positief is.

\section{BEWYS}

Vanuit

$$
\begin{aligned}
f(x \mid y)=x^{x} \quad e^{\frac{x x}{a r^{b}}} y & h_{x}\left(\frac{a}{\alpha}\right)^{x} \Gamma(\alpha) \\
& (x>0, y>0, a>0, \alpha>0),
\end{aligned}
$$

(3.1) en (3.2) volg dat

$$
\begin{aligned}
h(x)= & \frac{\mathrm{K} \cdot x^{\alpha}-1}{\Gamma(\alpha)\left(\frac{a}{\alpha}\right)^{\alpha}} \int_{0}^{x} y^{h z} e^{\frac{\alpha x}{a^{b}}} \\
& \cdot \mathrm{G}_{r, s}^{k, c}\left[\begin{array}{l}
\gamma y \mid \\
b_{1}, \ldots, b_{s}
\end{array}\right] d y .
\end{aligned}
$$

Beskou eerstens die integraal in (3.5) vir $h>0$.

Deur

(3.6) $e^{v z^{r}}=\mathrm{G}_{0.1}^{1.0}\left[\left.v z^{r}\right|_{0} ^{-}\right]$en

$$
\begin{aligned}
& \mathbf{G}_{r, s}^{h, r}\left[\begin{array}{l}
a_{1}, \ldots, a_{r} \\
b_{1}, \ldots, b_{s}
\end{array}\right]= \\
& \mathbf{G}_{s, r}^{t, k}\left[\begin{array}{cc}
1 & 1-b_{1}, \ldots, 1-b_{s} \\
z & 1-a_{1}, \ldots, 1-a_{r}
\end{array}\right]
\end{aligned}
$$

te gebruik, is

$$
\begin{aligned}
& 1=\int_{0}^{x} y, h x \mathrm{G}_{1.0}^{0.1}\left[\begin{array}{cc}
\frac{a y^{\prime \prime}}{x \alpha} & 1
\end{array}\right] \\
& \mathrm{G}_{r, s}^{\alpha,}\left[\begin{array}{cc}
a_{1}, \ldots, a_{r} \\
h_{1}, \ldots, h_{s}
\end{array}\right] d y
\end{aligned}
$$

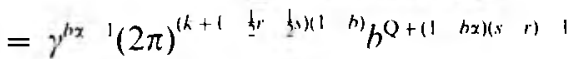

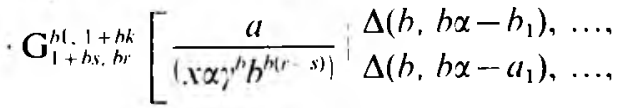

$$
\begin{aligned}
& \left.\begin{array}{l}
\Delta\left(b, b x-b_{s}\right), 1 \\
\Delta\left(b, b x-a_{s}\right)
\end{array}\right]
\end{aligned}
$$

(Mathai \& Saxena ${ }^{7}$, p. 80 )

en (3.3) volg duidelik.

Vir die geval $h<0$ is die integraal in (3.5) deur (3.6) te gebruik soos volg:

$$
\begin{aligned}
& 1=\int_{0}^{x} y^{b x} \mathrm{G}_{0.1}^{1.0}\left[\begin{array}{lll}
x x y y^{h} & - \\
a & 0
\end{array}\right] \\
& \cdot \mathbf{G}_{r, x}^{k \cdot 1}\left[\begin{array}{ll}
a_{1}, \ldots, a_{r} \\
b_{1}, \ldots, b_{s}
\end{array}\right] d y
\end{aligned}
$$

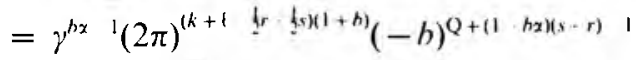

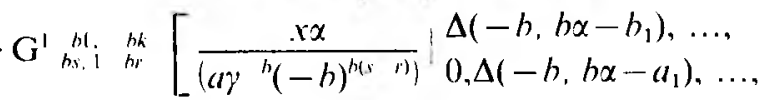

$$
\begin{aligned}
& \Delta\left(-b, b x-b_{s}\right) \\
& \left.\Delta\left(-b, b \alpha-a_{t}\right)\right]
\end{aligned}
$$

(Mathai \& Saxena7, p. 80).

Vanuit bostaande en (3.5) volg die onvoorwaardelike wdf. van $\mathrm{X}$ vir $b<0$ soos in (3.4) gegee.

\section{OPMERKING 3.1}

Tabel 1 toon enkele spesiale gevalle van hierdie algemene resultaat vir die tweeparametergammaverdeling as die voorwalardelike verdeling.

\section{OPMERKING 3.2}

Daar kan veronderstel word dat die voorwaardelike verdeling ' $\mathrm{H}$-funksieverdeling (kyk Springer'o, $\mathrm{p}$. 200 ) is, wat 'n veralgemening van die bekendste verdelings van nienegatiewe variate is. Veronderstel ook dat faktor $\mathrm{Y}$ ' $\mathrm{n} \mathrm{H}$-funksieverdeling besit en dat 'n algemene tipe regressiefunksie $a y^{b}$ van toepassing is. Bekker" (p. 91) definieer ' $n$ parameterryke onvoorwaardelike verdeling wat' $n$ wye klas van verdelings omsluit. Daar moet egter in gedagte gehou word wat die motivering vir hierdie ondersoek is, naamlik die verryking as gevolg van die regressieparameters $a$ en $b$ van die regressiefunksie $a y^{b}$.

\section{LITERATUURVERWYSINGS}

1. Roux, J. J. J., Bekker. A. \& Van der Merwe, C. A. (1989). Oor die onvoorwaardelike lognormaalverdeling, Die Suid-Afrikatase Tydskrif vir Natuurwetenskap en Tegnologie, 8, 144-148.

2. Erdélyi, A. et al. (1953). Higher transcendental functions, vol. 1 (McGraw-Hill, New York).

3. Erdélyi, A. et al. (1954a). Tables of integral transforms, vol. 1 (McGraw-Hill, New York). 
4. Bhattacharya, S. K. (1966). A modified Bessel function model in life testing. Metrika, vol. 11, 133-144.

5. Barr, A. \& Sichel, H. S. (1989). A note on some properties of compound poisson distributions (Research report, no. 2. Department of Statistics, University of South Africa, Pretoria).

6. Dubey, S. D. (1970). Compound gamma, beta and F distributions, Metrika, vol. 16, 27-31.

7. Mathai, A. M. \& Sixena, R. K. (1973). Generalized hypergeometric functions with applications in statistics and physical sciences (Springer-Verlag, Heidelberg).
8. Erdélyi, A et al. (1954b). Tables of integral transforms, vol. 2 (McGraw-Hill, New York).

9. Slater, L. J. (1960). Confluent hypergeometric lunctions (Cambridge University Press, London).

10. Springer, M.D. (1979). The algebra of random variables (Wiley, New York).

11. Bekker, A. (1990). Veralgemening, samestelling en karaklerisering as metodes om parameterryke verdelings te vind. Ph.D.proefskrif, Universiteit van Suid-Afrika. 